\title{
Spontaneous Intestinal Perforation is not Associated with the Recent Administration of Antenatal Betamethasone
}

\author{
Brian J Rau ${ }^{1}$, Marc G Weiss ${ }^{1}$, Jonathan K Muraskas ${ }^{1 *}$ and Carolyn Jones ${ }^{2}$ \\ ${ }^{1}$ Division of Neonatal-Perinatal Medicine, Maywood, Illinois, USA \\ ${ }^{2}$ Division of Genetics, Department of Pediatrics Loyola University Medical Center, Maywood, Illinois, USA
}

\begin{abstract}
Intestinal disease in premature infants, especially spontaneous intestinal perforation and necrotizing enterocolitis, contributes significant morbidity and mortality in very low birth weight infants and has a large impact in neonatal care and patient quality of life. In very low birth weight infants, factors influencing a systemic inflammatory response and/ or submucosal thinning, including postnatal glucocorticoids and exposure to indomethacin or ibuprofen, exacerbate the development of intestinal perforation in an already at risk population. In this retrospective analysis we evaluated whether antenatal steroids, a glucocorticoid for fetal lung maturity often given to mothers in close proximity to delivery, may be related to the development of perforation when given close to delivery and without adequate time for recovery of the intestinal mucosa. In our data set, it did not appear to be significantly related. Our results also did not show a categorical association between antenatal steroids and spontaneous intestinal perforation. We did however show a significant relationship between smaller, more depressed infants developing spontaneous intestinal perforation, as well as an association with concomitant sepsis.
\end{abstract}

Keywords: Spontaneous intestinal perforation; Necrotizing enterocolitis; Antenatal steroids; Betamethasone; Very low birth weight; Fetal infant

Abbreviations: SIP: Spontaneous Intestinal Perforation; NEC: Necrotizing Enterocolitis; ROP: Retinopathy of Prematurity; IVH: Intraventricular Hemorrhage; BPD: Bronchopulmonary Dysplasia; NRBC: Nucleated Red Blood Cell Count

\section{Brief Statement of Significance}

Intestinal disease in premature infants, especially spontaneous intestinal perforation and necrotizing enterocolitis, has been an intense focus of current and past research. The significant morbidity and mortality of these diseases creates a large impact in neonatal care and patient quality of life. In very low birth weight infants, factors influencing a systemic inflammatory response and/or submucosal thinning, including postnatal glucocorticoids and exposure to indomethacin or ibuprofen, exacerbate the development of intestinal perforation in an already at risk population. In this retrospective analysis we hypothesized that antenatal steroids, a glucocorticoid for fetal lung maturity often given to mothers in close proximity to delivery, may be related to the development of perforation when given close to delivery and without adequate time for recovery of the intestinal mucosa (Tables 1 and 2).

\section{Introduction}

Spontaneous Intestinal Perforation (SIP) is reported to occur in up to $7 \%$ of infants with a birth weight $<1000 \mathrm{~g}$ [1]. It is a condition that is pathologically dissimilar from Necrotizing Enterocolitis (NEC) and appears to be related to "skewed trophism" [2]. However,

\begin{tabular}{|c|c|c|c|}
\hline Variable & SIP $(N=17)$ & Control $(\mathrm{N}=60)$ & p Value \\
\hline $\begin{array}{l}\text { Antenatal Steroids prior to } \\
\text { birth (hours) }\end{array}$ & $32(3,240) N=14$ & $51(1,696), N=52$ & NS \\
\hline Gestational Age (weeks) ${ }^{b}$ & $25.1(23.2,28.20) \mathrm{N}=17$ & $27.2(22.0,35.1) \mathrm{N}=60$ & 0.0004 \\
\hline Birth Weight $(\mathrm{g})^{\mathrm{b}}$ & $697(300,1272) \mathrm{N}=17$ & $889(530,1260) \mathrm{N}=60$ & 0.002 \\
\hline NRBC at birth ${ }^{a}$ & $15(2,220) \mathrm{N}=17$ & $21(2,996), N=60$ & NS \\
\hline Apgar at 5 minutes ${ }^{a}$ & $7(0,9) \mathrm{N}=17$ & $8(3,9), N=60$ & 0.04 \\
\hline
\end{tabular}

Abbreviations: NRBC, nucleated red blood cells; NS, non significant

${ }^{\text {a }}$ Median, range

${ }^{\mathrm{b}}$ Mean, range

Table 1: Comparison by Group. both SIP and NEC are associated with significantly greater morbidity and mortality than in infants without these diseases. NEC often begins with a necrotic segment of small intestine, gas accumulation in the submucosa of the bowel wall known as pneumatosis, followed by perforation of the bowel. Most of these infants were previously fed enterally, and generally occurs at two to six weeks of life. As compared to infants with NEC, infants with SIP have been described as having an earlier onset of disease with the main occurrence prior to two weeks of life, a gasless abdomen, a location within the terminal ileum, and absence of pneumatosis [3]. Pathogenic factors in SIP are not well understood. It has been recognized that SIP is due to a deficiency of the enteric musculature, specifically the muscularispropria [4]. There is no inflammation and no evidence of ischemia.

In a neonatal mouse model, dexamethasone caused submucosal thinning and mucosal hyperplasia within the ileum [5]. In a large cohort of infants with SIP (633 patients), extremely low birth weight infants were more likely to acquire perforation after combined exposure to indomethacin and glucocorticoids (either created endogenously through stress or given exogenously) [6,7]. Although

\begin{tabular}{|l|l|l|}
\hline Variable & SIP $(\mathbf{N}=17)$ & Control $(\mathbf{N}=\mathbf{6 0})$ \\
\hline Death & $11.8 \%(n=2)$ & $0.02 \%(n=1)$ \\
\hline IVH (grade 3 and 4) & $41.2 \%(n=7)$ & $18.3 \%(n=11)$ \\
\hline ROP (stage 2 and 3) & $64.7 \%(n=11)$ & $38.3 \%(n=23)$ \\
\hline BPD & $70.6 \%(n=12)$ & $43.3 \%(n=26)$ \\
\hline
\end{tabular}

Table 2: Outcomes by Group.

*Corresponding author: Jonathan K Muraskas, MD, Division of Neonatal-Perinatal Medicine, Loyola University Medical Center, 2160 S. 1st Avenue, 107-5810, Maywood IL 60153, USA, Tel: (708) 216-1067; Fax: (708) 216-5602; E-mail: jmurask@lumc.edu

Received June 14, 2012; Accepted November 23, 2012; Published November 26, 2012

Citation: Rau BJ, Weiss MG, Muraskas JK, Jones C (2013) Spontaneous Intestinal Perforation is not Associated with the Recent Administration of Antenatal Betamethasone. J Neonatal Biol 2:112. doi:10.4172/2167-0897.1000112

Copyright: (c) 2013 Rau BJ, et al. This is an open-access article distributed under the terms of the Creative Commons Attribution License, which permits unrestricted use distribution, and reproduction in any medium, provided the original author and source are credited. 
several neonatal risk factors have been associated with SIP, little is known about prenatal risk factors. Antenatal indomethacin for tocolysis has been implicated in increasing the risk for periventricular leukomalacia and NEC in premature infants [8]. SIP has also been related to placental inflammation, [9] as well as systemic Candida fungal infection. In 2006, Attridge et al. performed a retrospective analysis of 388 infants with SIP and 388 control infants, evaluating for multiple associated variables [10]. They did not find an association between the presence and absence of antenatal steroids.

In this retrospective analysis, we hypothesized that antenatal steroids may be related to the development of perforation when given close to delivery and without adequate time for recovery of the intestinal mucosa.

\section{Methods}

This study is a retrospective analysis of very low birth weight infants (birth weight $\leq 1500 \mathrm{~g}$ ) who were taken care of at a single institution between August 2006 and December 2011. The study protocol was approved by the institutional review board of Loyola University Medical Center. Our database was queried to identify all infants with SIP. A random sequential number generator was used to identify a cohort of infants $\leq 1500 \mathrm{~g}$ without SIP who were born during the same epoch.The medical charts were reviewed for neonatal demographic data, Apgar score at 5 minutes, Nucleated Red Blood Cell Count (NRBC) at birth, blood culture results 48 hours before and after occurrence of SIP, Bronchopulmonary Dysplasia (BPD) defined as oxygen requirement at 36 weeks adjusted gestation, Intraventricular Hemorrhage (IVH), Retinopathy of Prematurity (ROP), as well as the administration of indomethacin, ibuprofen, or postnatal steroids within the first 14 days of life. An infant was considered to have SIP if they had an absence of pneumatosis on radiography, were $<3$ weeks old, and no surgical or histopathological findings of NEC. Antenatal data was collected on maternal demographics, placental histopathology, and steroid administration prior to delivery. At our institution, betamethasone $12 \mathrm{mg}$ IM every 24 hours is the standard steroid regimen given to mothers with threatened premature birth.

Data were analyzed using SAS version 9.1 (SAS Institute Inc., Cary, NC, USA). Wilcoxon rank sum tests and two sample $t$-tests were used to examine if there is significant difference in continuous variables between cases and controls. Fisher's exact tests were used to examine the association between the group and categorical variables.

\section{Results}

Of the infants at our institution who developed SIP during the time period between August 2006 and December 2011, we identified 17 with a birth weight $\leq 1500 \mathrm{~g}$. A randomized cohort of 60 infants $\leq 1500 \mathrm{~g}$ without SIP was also identified. Of the infants with SIP, three (18\%) did not receive antenatal steroids versus eight (13\%) in the control group. There was no significant difference between these two groups using Fisher's exact tests $(p=0.7)$. There was also no significant difference when comparing the timing of antenatal steroid administration prior to delivery $(p=0.25)$. Mothers of infants who later developed SIP received steroids a median of 32 hours prior to delivery (range 3-240 hours). The control group received steroids approximately 51 hours prior to delivery (range 1-696 hours). Two patients had blood cultures positive for Candida albicans in the 48 hours prior to perforation, two had coagulase negative Staphylococcus sepsis, and one patient had extended spectrum beta-lactamase positive Escherichia coli sepsis. No positive blood cultures were obtained in the control group. There was no placental histopathology available on any patient in the study.
No difference in NRBC count was significant between the two groups (median 15 (case) vs 21 (control)).

Table 1 shows the comparison between infants with SIP and control infants. Infants who later developed SIP were significantly more likely to be of younger gestational age $(\mathrm{p}=0.0004)$ with a mean gestational age of 25.1 weeks vs. 27.2 weeks in the controls. There was one outlier in the control group with a gestational age of 35.1 weeks. No difference was significant between genders in the two groups. The case group contained seven females and 10 males while the control group contained 30 females and 30 males. Twins were prevalent in both groups with $41 \%(n=7)$ in cases and $27 \%(n=16)$ in controls. There was no case of both twins developing SIP. Infants with SIP were also smaller with a mean weight of 697 grams vs. 889 grams $(p=0.02)$, and were more depressed at birth with a 5 minute Apgar median of 7 (range $0-9)$ vs. 8 (range 3-9) in controls $(\mathrm{p}=0.04)$. All infants were $<1300 \mathrm{~g}$ at birth. No difference was significant between the groups when comparing postnatal steroid administration $(\mathrm{p}=0.08)$, although there was a trend towards infants with SIP receiving postnatal steroids earlier in life vs. control infants. Six infants (35\%) in the case group received postnatal steroids (five within 48 hours of birth and one 10 days after birth). Of the five infants with very early steroid administration, they received hydrocortisone $3 \mathrm{mg} / \mathrm{kg} / \mathrm{day}$. The one infant with steroids on day of life 10 received dexamethasone $0.5 \mathrm{mg} /$ $\mathrm{kg} /$ day. Nine patients (15\%) in the control group received postnatal steroids in the first one to two weeks of life. More infants with SIP appeared to receive ibuprofen for patent ductus arteriosus than indomethacin vs. the control group $(41 \%(n=7)$ vs. $18 \%(n=11))$, but we did not see a statistically significant difference between the two groups $(\mathrm{p}=0.10)$. Rates of postnatal indomethacin exposure were similar $(12 \%(\mathrm{n}=2)$ vs. $15 \%(\mathrm{n}=9))(\mathrm{p}=1.0)$. Table 2 denotes outcomes for these infants by time of discharge.

\section{Discussion}

Intestinal disease in premature infants, especially spontaneous intestinal perforation and necrotizing enterocolitis, has been an intense focus of current and past research. The significant morbidity and mortality of these diseases creates a large impact in neonatal care and patient quality of life. Definitive prevention of SIP and NEC has been elusive. Our understanding of SIP has been growing and many pathophysiological changes have been identified. With SIP appearing to be related to "skewed trophism" and deficiencies of the muscularispropria $[2,4]$, there exists debate as to whether these changes are acquired, innate, or both. In very low birth weight infants, factors influencing a systemic inflammatory response and/ or submucosal thinning, including postnatal glucocorticoids and exposure to indomethacin or ibuprofen, exacerbate the development of intestinal perforation in an already at risk population.

It can be postulated that antenatal steroids, as glucocorticoids, would theoretically contribute to this risk. They have a high rate of administration in the at-risk group and are often given in close proximity to delivery. It has previously been suggested in a large cohort analysis that there is categorically no association between antenatal steroids and SIP. Our hypothesis was that antenatal steroids may be related to the development of perforation when given close to delivery and without adequate time for recovery of the intestinal mucosa. In our small data set, it did not appear to be significantly related. It is possible that a larger case sample would further delineate the relationship, but we presume the effect would be small.

Levels of glucocorticoid bioactivity have been shown to be 
Citation: Rau BJ, Weiss MG, Muraskas JK, Jones C (2013) Spontaneous Intestinal Perforation is not Associated with the Recent Administration of Antenatal Betamethasone. J Neonatal Biol 2:112. doi:10.4172/2167-0897.1000112

elevated $>4$ fold in newborns that were born $<12$ hours after the dose of betamethasone was given, a response that resolved if three days passed between steroid dose and delivery [11]. Five of the infants in our case sample received hydrocortisone within 48 hours after delivery, but on further analysis these infants were exposed to antenatal betamethasonebetween 48-240 hours prior to delivery. Our results also did not show a categorical association between antenatal steroids and SIP, in agreement with Attridge et al. We did however have a significant relationship between smaller, more depressed infants developing SIP, as well as an association with concomitant sepsis. With overwhelming evidence that antenatal steroids reduce the risk of neonatal mortality, RDS, and IVH, the benefits of this medication continue to outweigh the risks [12]. Future research will hopefully further elucidate the pathophysiological triggers for SIP and its avoidance.

\section{Acknowledgements}

We gratefully acknowledge the assistance and support of Mrs. Bonnie Kanzia in the Division of Neonatal-Perinatal Medicine, and Rong Guo in the Research Services Department.

\section{References}

1. Uceda JE, Laos CA, Kolni HW, Klein AM (1995) Intestinal perforations in infants with a very low birth weight: a disease of increasing survival? J Pediatr Surg 30: 1314-1316.

2. Gordon PV (2009) Understanding intestinal vulnerability to perforation in the extremely low birth weight infant. Pediatr Res 65: 138-144.

3. Pumberger W, Mayr M, Kohlhauser C, Weninger M (2002) Spontaneous localized intestinal perforation in very-low-birth-weight infants: a distinct clinical entity different from necrotizing enterocolitis. J Am Coll Surg 195 796-803.
4. Holland AJ, Shun A, Martin HC, Cooke-Yarborough C, Holland J (2003) Small bowel perforation in the premature neonate: congenital or acquired? Pediatr Surg Int 19: 489-494.

5. Gordon PV, Price WA, Stiles AD (2001) Dexamethasone administration to newborn mice alters mucosal and muscular morphology in the ileum and modulates IGF-I localization. Pediatr Res 49: 93-100.

6. Attridge JT, Clark R, Walker MW, Gordon PV (2006) New insights into spontaneous intestinal perforation using a national data set: (2) two populations of patients with perforations. J Perinatol 26: 185-188.

7. Paquette L, Friedlich P, Ramanathan R, Seri I (2006) Concurrent use of indomethacin and dexamethasone increases the risk of spontaneous intestinal perforation in very low birth weight neonates. J Perinatol 26: 486492.

8. Amin SB, Sinkin RA, Glantz JC (2007) Metaanalysis of the effect of antenata indomethacin on neonatal outcomes. Am J Obstet Gynecol 197: 486.e1-486. e10.

9. Ragouilliaux CJ, Keeney SE, Hawkins HK, Rowen JL (2007) Maternal factors in extremely low birth weight infants who develop spontaneous intestina perforation. Pediatrics 120: e1458-e1464.

10. Attridge JT, Clark R, Gordon PV (2006) New insights into spontaneous intestinal perforation using a national data set (3): antenatal steroids have no adverse association with spontaneous intestinal perforation. J Perinatol 26: $667-670$.

11. Kajantie E, Raivio T, Janne OA, Hovi P, Dunkel L, et al. (2004) Circulating glucocorticoid bioactivity in the preterm newborn after antenatal betamethasone treatment. J Clin Endocrinol Metab 89: 3999-4003.

12. The Effect of Corticosteroids for Fetal Maturation on Perinatal Outcomes. National Institutes of Health Consensus Development Conference Statement 1994 Feb 28-Mar 2 